\title{
Sistema de orientación vocacional profesional en la construcción de proyectos de vida
}

\section{Professional vocational guidance system in the construction of life projects}

DOI: $10.46932 / \mathrm{sfjdv2n2-178}$

Received in: March 1st, 2021

Accepted in: May 30th, 2021

\author{
Kerly Leomar Toala Vera \\ Licenciada en Ciencias de la Educación especialización Historia y Geografía \\ Magister en Docencia e Investigación Educativa \\ Analista del Centro de Promoción y Apoyo al Ingreso \\ Universidad Técnica de Manabí \\ E-mail: kerly.toala@utm.edu.ec; kerlyta13@hotmail.es
}

\section{Francisco Antonio Mawyin Cevallos}

Licenciado en Ciencias de la Educación especialidad Psicología y Orientación Vocacional

Magister en Orientación Educativa Vocacional y Profesional

Doctor Dentro del Programa de Doctorado en Educación

Docente Auxiliar Tiempo Completo

Universidad Técnica de Manabí

E-mail: franciscomawyin2016@gmail.com; francisco.mawyin@utm.edu.ec

\section{Gustavo Adolfo Santana Sardi}

Licenciado en Ciencias de la Educación Técnica mención Contabilidad Computarizada

Diplomado en Desarrollo de Estrategias Digitales de Aprendizaje

Magister en Gerencia Educativa

Analista del Centro de Promoción y Apoyo al Ingreso

Universidad Técnica de Manabí

E-mail: gusansar@hotmail.com,gusansar@gmail.com,gustavo.santana@utm.edu.ec

Pedro Alejandro Escobar Palma

Licenciado en Ciencias de la Comunicación mención Periodismo

Magister en Periodismo mención en Periodismo Digital y Gestión de Proyectos Multimedia

Periodista del departamento de redacción

Medios Ediasa

E-mail: pedroescohbk@gmail.com; pescobar@eldiario.ec

\section{RESUMEN}

La siguiente investigación se basa en un sistema de orientación vocacional profesional en la construcción de proyectos de vida y para hablar sobre la orientación vocacional y profesional es indispensable adentrarse en el objeto de estudio y para esto se logra desarrollar un camino desde el inicio y todo el progreso de este campo, el concepto de orientación vocacional y profesional parte desde el momento en que el hombre en su necesidad de conformar la sociedad, se da cuenta y reconoce que de acuerdo al rol que desempeña cada individuo cumple un papel fundamental en la misma. La orientación vocacional profesional es un apoyo al proceso de la toma decisiones como parte de un proyecto de vida, en él, el individuo conoce sus capacidades, potencialidades, intereses y limitaciones o dificultades, asî como sus posibilidades existentes en el medio que se desarrolla. El proceso de construcción de proyectos 
de vida requiere de un enfoque causal, donde es necesario el análisis individualizado, contextualizado y dinámico de cada persona. Este estudio es realizado bajo la técnica documental tipo bibliográfico el cual permitió una investigación sobre las posibles necesidades de cada individuo para su adecuada estructuración y la elaboración de un sistema de orientación vocacional profesional con un enfoque individualizado, contextualizado del proceso, lo que conllevó al esclarecimiento de las metas futuras de cada persona y regular de manera efectiva sus conductas para el logro de las metas trazadas.

Palabras claves: Orientación vocacional profesional, proyecto de vida, procesos, sistemas.

\begin{abstract}
The following research is based on a system of professional vocational guidance in the construction of life projects and to talk about vocational and professional guidance it is essential to delve into the object of study and for this it is possible to develop a path from the beginning and all the progress in this field, the concept of vocational and professional orientation starts from the moment that man in his need to shape society, realizes and recognizes that according to the role that each individual plays a fundamental role in it. Professional vocational guidance is a support to the decision-making process as part of a life project, in it, the individual knows their capacities, potentialities, interests and limitations or difficulties, as well as their existing possibilities in the environment that develops. The process of building life projects requires a causal approach, where an individualized, contextualized and dynamic analysis of each person is necessary. This study is carried out under the bibliographic documentary technique which allowed an investigation on the possible needs of each individual for their adequate structuring and the elaboration of a professional vocational guidance system with an individualized, contextualized approach to the process, which led to the clarification of the future goals of each person and effectively regulate their behaviors to achieve the goals set.
\end{abstract}

Keywords: Professional vocational guidance, life project, processes, systems.

\title{
1 INTRODUCCION
}

La Orientación Vocacional fue determinada como un proceso de ayuda para la elección y desarrollo profesional, pero dicho valor se limitó al momento de la elección de una profesión. La OV en el tiempo ha estado influenciada por fundamentos filosóficos, sociológicos, psicopedagógicos y tecnológicos hacia una orientación profesional efectiva que coadyuve al diseño de un sistema que integre la relación escuela-familia-comunidad a partir de la construcción de proyectos de vida.

Fitch (citado en Maura, 2003, p. 260) definió la OV como: El proceso de asistencia individual para la selección de una ocupación, preparación para la misma, inicio y desarrollo en ella. Al mismo tiempo emergieron las teorías económicas que, contrario a las posiciones al azar, ven a los sujetos al margen de lo que aspiran por ellos mismos. La orientación vocacional se analiza en la complejidad de la naturaleza de la motivación humana y su función reguladora en la toma de decisiones con relación a una profesión desde concepciones funcionalistas, psicoanalíticas y humanistas, ya que para ello se parte del reconocimiento de la unidad dialéctica entre la naturaleza subjetiva, activa de la psiquis y su determinación histórico-social, lo que condiciona cualquier posición asumida. En este contexto, cabe 
citarse lo mencionado por Álvarez de Sayas (2016), quien sostiene que el principio de que el hombre se forma para la vida parte de reconocer todas las acciones formativas desde la escuela hasta la propia convivencia social que tienen lugar en la preparación para una profesión.

A lo largo de los años las tendencias en orientación vocacional profesional (OVP) se han centrado en intervenciones individuales con un carácter reactivo y terapéutico, enfocadas a la resolución de problemas puntuales y teniendo poco en cuenta el contexto.

Cabe destacar que la práctica de la OVP en Ecuador es un gran reto, ya que en el país se configura un escenario de diversidad cultural, con características heterogéneas de los actores en diversos contextos culturales.

En el Ecuador, la práctica orientativa se ha circunscrito a la educación media, en donde su aplicabilidad ha sido limitada por la ausencia de herramientas psicométricas adaptadas al contexto nacional, el desconocimiento de las fortalezas que tiene en la construcción de factores protectores en niños, niñas y adolescentes, la invisibilidad histórica que ha tenido esta disciplina en el país. En 2018, el mismo Ministerio de Educación puso a disposición del sistema educativo el documento Herramientas para orientar la construcción de proyectos de vida de estudiantes. Esta publicación presenta un sinnúmero de actividades lúdicas y reflexivas orientadas al acompañamiento en la construcción de proyectos de vida de niños, niñas y adolescentes y personas adultas (Ministerio de Educación del Ecuador, 2018).

El presente documento, examina el sistema de orientación vocacional profesional y las condiciones psicosociales que sustentan el análisis de la OVP y la construcción de un proyecto de vida. A continuación, el proceso para investigar el Sistema de Orientación Vocacional y Profesional se realiza. También se describen los métodos para su investigación y algunos resultados previos que permiten establecer una lista de recomendaciones a considerar por parte de las personas para obtener una buena construcción de proyecto de vida.

\section{DESARROLLO}

Como ocurre con cualquier otra disciplina de reciente instrumentación pero remota en su aplicación, llegar a una conceptualización universalmente asentada de la orientación, ha tomado largos tramos históricos caracterizados por la dificultad en la delimitación de los principios, alcances, funciones, objetivos, modelos, áreas, métodos empleados, contextos de intervención y la definición de sus agentes. Lo que siempre ha estado claro es la vinculación entre enseñanza y orientación, siendo la primera el principio que estimula la capacidad de conocer, mientras que la segunda estimula la capacidad de decidir (Martín, 1996). El concepto de la orientación fue constituyéndose en un constructo que respondía a los diversos modelos teóricos y enfoques del desarrollo humano. Las teorías humanistas destacan el papel 
protagónico de la persona con base en su auto conocimiento. De acuerdo a esto, los enfoques evolucionistas reconocen a la vocación como una expresión de la personalidad, mientras que el enfoque histórico-cultural conceptualiza la orientación como un proceso dialéctico que responde a elementos internos de la persona, los cuales que están mediados por lo social (Carmona, Cortina, \& Diana, 2012).

Un aspecto relevante es que dentro de las funciones a cumplir por las personas, consta el trabajo en procesos de orientación vocacional y profesional con miras a construir proyectos de vida sustentados en el autoconocimiento, la información y la toma de decisiones, bajo un enfoque de derechos (Ministerio de Educación del Ecuador, 2016). Un conjunto de acciones de acompañamiento (educativopsicológicosocial) y asesoramiento (individual y grupal) dirigido tanto a estudiantes como a personas para que, de manera individual y con base en el autoconocimiento y la información disponible, tomen decisiones vocacionales y profesionales adecuadas como parte de la construcción de su proyecto de vida" (Ministerio de Educación del Ecuador, 2015), esto hace énfasis en la importancia de reconocer los factores internos y externos que influyen en la construcción de identidades vocacionales, mediante procesos de autoconocimiento, información y toma de decisiones.

Sobre la base del recorrido histórico anteriormente expuesto, es loable hacer énfasis en que la orientación ha experimentado un cambio estructural en su abordaje conceptual, dejando de centrarse exclusivamente en los procesos laborales e intereses profesionales para enfocar su abordaje desde la preocupación por las trayectorias vitales de niños, niñas y adolescentes más allá de su desarrollo profesional (Donoso-Vázquez \& Sánchez Martí, 2013). Con este cambio de enfoque queda en evidencia la importancia de la orientación en el reconocimiento de las personas como actores sociales que construyen su propia identidad y, a través de este proceso, contribuyen a la construcción de la sociedad.

El proceso de orientación vocacional y profesional es pilar fundamental para llegar a la elección correcta del futuro de la persona; por ende, tiene que ser planificado, programado, ejecutado y evaluado JARRIN, P. (2002). Existen dos clases de factores que inciden en el mismo: Factores internos y externos.

Factores Internos: Forman parte de la personalidad del ser humano ayudan a descubrir cuál es la: inclinación, afición, interés, vocación, actitud y aptitud del educando frente a una profesión y ocupación FELDMAN, R. (2007). Para que estos factores sean descubiertos, tienen que recurrir a la aplicación de técnicas personalizadas a la realidad vocacional, entrevistas, ejercicios de dinámica de grupos, ejercicios vivenciales. Dan la pauta para descubrir los intereses vocacionales y puedan proseguir las carreras universitarias.

Factores Externos: Tienen gran importancia en el proceso de Orientación vocacional y profesional, así tenemos la familia y el medio donde se desarrolla. 
- La familia tiene injerencia directa y determinante en el futuro profesional; por ejemplo en la profesión que tienen sus padres, cuya influencia es positiva en cuanto se refiere a intereses, habilidades y vocaciones pre-profesionales y negativas cuando "obligan" a sus hijos en lo que ellos conocen como tradición familiar. Se conocen familias de abogados, médicos, profesores, mecánicos, carpinteros, que ya tienen instrumentos y aparatos necesarios que les facilitará una eficaz y rápida profesionalización.

- El medio (conformado por compañeros, amigos, colegio, nuevas tecnologías, entre otros), también influye directamente en la elección profesional. Siendo un largo proceso de descubrimiento y aprendizaje de intereses, habilidades y vocaciones, es importante la ayuda de un equipo multidisciplinario para redescubrir las competencias o su nivel de desempeño en cada área del conocimiento LEVIS, R. (2003).

Esto les permite apoyar y reforzar sus propios comportamientos y los de su grupo o comunidad, frente a ello, propone las siguientes condiciones para favorecer el fortalecimiento personal:

- Colaboración para aislar los problemas y establecer un plan de actuación

- Reconocimiento de factores del contexto que impiden el fortalecimiento personal (pobreza, marginación, sexismo, racismo, etc.) y, por ende, la superación de los problemas

- Conocimiento crítico para definir un problema, organizar la información y proponer soluciones

- Competencia necesaria para la resolución de los problemas

- Creación de comunidad entre quienes comparten unos mismos objetivos.

Bajo esta perspectiva, el rol del orientador adquiere una connotación activa de agente social, por lo que se le atribuye la responsabilidad.

Los proyectos de vida son el resultado de la conciliación entre la toma de decisiones racional y emocional. La toma de decisiones no solo se debe a un proceso puramente cognitivo, sino que está fuertemente influenciada por las condiciones inter e intrapersonales de cada individuo: emociones, sentimientos, temperamento, intereses, etc. La capacidad de proyectar nuestra vida en el futuro permite a las personas elaborar metas y objetivos en el ámbito personal, social, cultural, laboral, moral, etc. (Lomelí-Parga, López-Padilla \& Valenzuela-González, 2016; Melendro, 2014; Pérez, Poza \& Fernández, 2016; Pérez \& Melendro, 2016; Rodríguez, Juanas \& González, 2016) p. 150.

Tener un proyecto vital sólido y viable es complicado, pero absolutamente necesario si se quiere manejar las complejidades de las trayectorias vitales y las transiciones relacionadas con la carrera (Maree, 2018) p. 150. Queda claro que el proyecto de vida es, sin lugar a duda, un proceso que constituye una directriz o guía de las motivaciones, intereses y expectativas de cada individuo frente a la definición de certezas futuras. 
La humanidad vive bajo el imperio de la caducidad. Cambian las formas de percibir el mundo, la felicidad, el amor, las relaciones sociales, el rol del trabajador, lo placentero, la proyección de vida. La modernidad líquida entonces, se caracteriza por una inestabilidad que se asocia a la desaparición de referentes a los que anclar las certezas. La sociedad moderna, vista solo como sociedad de trabajo, aniquila toda posibilidad de acción, degradando al ser humano a animal laboran, a meros animales trabajadores (Chul Han, 2017), que construyen su proyecto de vida en relación a cumplir solo dicha función: trabajar para alcanzar. Frente a este escenario nada estimulante de incertidumbres, la definición de un proyecto de vida se constituye en un ejercicio complejo de proyección futura. Es allí en donde la orientación vocacional debe construir certezas frente a un mundo incierto. Para ello, debe utilizar herramientas de autoconocimiento que permitan conectar nuevamente a los sujetos a una visión integrada al todo.

La orientación desde esta perspectiva es entendida como educación vocacional, basada en la investigación activa por parte del alumno para el desarrollo de su propio proyecto de vida. Para que el joven enfoque su futuro personal, social y laboral, se utiliza un método que articula la reflexión con pares y adultos, la investigación y la acción, para desarrollar los recursos personales en un aprendizaje activo y de experiencia personal. Esta nueva perspectiva para la orientación trata de promover una revisión de la creencia del "yo quiero realizarme plenamente" ya que la misma podría conllevar la destrucción de todo lo que se perciba como un obstáculo para dicha realización personal. Todo, incluyendo al otro. Por su parte, la elección vocacional es tanto un proceso como un acto de elegir objetos (a los que convencionalmente definimos como vocacionales). Es un proceso ya que se trata de un trayecto o itinerario de vida inacabado, a través del cual el sujeto reconoce y encuentra dentro de una gran variedad uno o más objetos con los que establece vínculos singulares. El proceso supone la construcción y reconstrucción histórica de los vínculos actuales y pasados, al tiempo que la imaginación de los futuros. El proceso y el acto de elegir tienen aspectos conscientes e inconscientes. En la búsqueda incesante que implica la elección de un objeto vocacional hay momentos de enamoramiento, de ilusión, de euforia, pero también de desencanto, de desilusión, de apatía.

Desde la orientación, la transición se concibe como una oportunidad de desarrollo personal, a través de la cual, la persona debe aprender a gestionar e integrar los cambios que se producen en él mismo y en su entorno con la pretensión de construir, como agente activo, su propio proyecto vital y así, planificar y afianzar su desarrollo personal y profesional, además de ajustarse a la rapidez y vertiginosidad de los cambios que acontecen en la sociedad y que operan en el ámbito social, formativo y laboral. (González y González, 2015, p. 30).

Según ( MINEDUC, 2015) para poder trabajar el tema de la OVP, es importante que partamos de una definición general sobre este concepto: La orientación vocacional y profesional (OVP) comprende 
un conjunto de acciones de acompañamiento (educativo-psicológico-social) y asesoramiento (individual y grupal) dirigido a las y los estudiantes de una institución educativa para que, de manera individual y con base en el autoconocimiento y la información disponible, tomen decisiones vocacionales y profesionales adecuadas como parte de la construcción de su proyecto de vida, la educación es uno de los espacios más importantes y decisivos de formación vocacional y profesional en donde las y los estudiantes contarán con un sinnúmero de estímulos y experiencias que les permitirán explorar sus gustos, habilidades, intereses, capacidades y deseos en relación con lo que quieran hacer en la vida. Según menciona (Santana Sardi y Vigueras Moreno ,2019; p. 902) la creación de las bases económico-sociales y las exigencias actuales de su desarrollo, requieren continuar elevando la calidad del trabajo educacional, en el cual constituye un punto importante el desarrollo de intereses en las personas. De esta manera, la construcción del proyecto de vida en los individuos se basa en las experiencias vividas en su entorno, dando apertura a su conocimiento para saber lo que realmente quiere en su diario vivir.

Como lo menciona en su artículo (Alcívar, Santana, Zambrano, Sardi, \& Riverón, 2020; p. 911), Para la orientación profesional se conocen diferentes métodos que concretan su desarrollo en el proceso formativo, producto a que estos "(...) son las vías que se siguen para lograr el objetivo educativo planteado por la sociedad y las tareas específicas a desarrollar en condiciones determinadas por el proceso educativo."

La construcción de un proyecto de vida, según J. R. Ruiz, (2011), llega a implicar el reconocimiento de las condiciones de la persona desde su entorno, sus relaciones familiares y sociales, con lo cual se debe modelar un ejercicio racional de toma de decisiones bajo una motivación permanente y que da origen a la planeación consciente en el tiempo desde las dimensiones laborales, sociales y/o profesionales, entre otras, con la necesidad de llevar un registro frente a lo planeado. Existen varias formas de llegar a influenciar a una persona a realizar cambios en su proyecto de vida, muchas de ellas llegan a suceder por la influencia de su entorno, sea familiar o adentro del círculo de amistades. Por esto se necesita hacer entender a las personas en general sobre la inclusión de todo tipo de personas, sea con algún problema físico o mental, en la educación sobre los proyectos de vida.

De las diversas teorías que giran en torno a lo que es el proyecto de vida, los autores Moje Mayorca \& Woolcott Oyague (2018) detallan sobre la teoría del daño al proyecto de vida el cual: “... tiene como sustento original la filosofía de la existencia, la cual postula que la libertad compone la esencia misma del hombre, cualidad que lo hace diferente y único ante los demás seres de la naturaleza y le concede dignidad.

Facultad que permite que cada individuo decidir y elegir después de valorar lo que se denomina "proyecto de vida" o proyecto existencial.", dicho de otra manera, proyecto de vida es el ejercicio de idealizar la vida, dibujando el futuro que se desea de forma anticipada a fin de completarse 
uno como persona, darle una razón de ser a su existencia y de transcendencia al vivir. Con esta premisa es inimaginable como resultará el proyecto de vida, y consecuentemente sin una certeza del éxito o fracaso por parte de cada persona. Por esto, los seres humanos tienen el privilegio de decidir y/o elegir de forma libre e individual un proyecto de vida, intentando múltiples maneras de realizarlo.

No solo aquellos que pertenecen al ámbito educativo influyen en la realización del proyecto de vida de una persona sino también dentro del ámbito familiar suele suceder, independientemente si la persona está cruzando ya la vida universitaria, los propios miembros de la familia llegan a influenciar a los planes de vida, hasta el punto en el que llega a ser completamente diferente de lo que había pensado por uno mismo. Es necesario tener en cuenta las palabras que Gallegos Navas (2017) menciona: "Es importante analizar que cada familia tiene su estilo de vida y circunstancias que la condicionan... Es importante recalcar que el estilo de vida contribuye a la conformación de la identidad familiar, de ahí que el modo de vida de las personas esté determinado por el estilo de vida de su familia”, (Gallegos Navas, 2017), aclarando que cada persona tiene una diferente perspectiva y diferentes planes en su proyecto de vida, varios de ellos siendo incentivados por los padres en que, sigan los propios planes de la persona demostrando parcial o total apoyo, o por otra parte, convencer a la persona para que cambie sus planes a los que la propia familia desea para él.(Flores Delgado, 2021).

En el país Ecuador, antes de concluir los estudios del bachillerato, deben de pasar por un sistema de evaluación y orientación de sus intereses y aptitudes vocacionales como parte del programa OVP (Orientación Vocacional y Profesional) que tiene como objetivo encaminar a los adolescentes en la vida universitaria para posterior ser parte de la matriz productiva, (Gómez \& Palma, 2019), por ende, actualmente es necesario hacer pensar a aquellos jóvenes que es lo que desean realizar durante su vida universitaria y, a futuro, su vida laboral.

Además, según diversas indagaciones que Mendoza (2016) realiza acerca de las conceptualizaciones y/o prácticas en algunos países latinoamericanos, permite comprimirlas en las siguientes tendencias acerca de la elaboración de proyectos personales de vida como vía para la orientación vocacional:

1. Es necesario un medio de preparación para el trabajo y la formación calificada para ejercerlo y proceso de autodeterminación y la proyección futura de la personalidad, en base a la capacidad de auto dirigir su vocación de autorrealización.

2. Desarrollar un proyecto de vida viable y comprometido con la realización personal a corto, mediano y largo plazo desde una perspectiva general, axiológica e integral para forjar identidades a partir de la articulación del pasado, presente y futuro en la construcción de un sujeto 
no solo histórico, sino social y por supuesto político y ciudadano donde coloca en juego dinámico su individualidad en los diferentes contextos. (Mendoza et al., 2016).

Durante el transcurso de los últimos años, el Ministerio de Educación del Ecuador ha emitido varios documentos que permiten que sirven como manuales o herramientas para los docentes de las

instituciones educativas, enfocados en la educación secundaria y de bachillerato, así, desde el 2015 el ministerio empezó a emitir el documento de Manual de Orientación Vocacional y Profesional para los Departamentos de Consejería Estudiantil el cual provee a los estudiantes un conjunto de acciones de acompañamiento (educativo-psicológico-social) y asesoramiento (individual y grupal) para la toma de decisiones vocacionales y profesionales adecuadas para estos. Después, en 2017 se emitió el documento de Manual de interpretación del Inventario de Preferencias Profesionales para Jóvenes IPPJ en donde se provee un cuestionario psicométrico. Todos estos recursos son indispensable para una buena orientación vocacional para poder construir un buen proyecto de vida.

\section{CONCLUSIÓN}

Los resultados presentados en el presente documento son preliminares, ya que son parte de una investigación bibliográfica. Sin embargo, los resultados obtenidos demuestran un efecto positivo reflejado en las teorías de distintos autores desde diferentes puntos de vista.

Se pudo recabar que las tendencias en orientación vocacional se han centrado en intervenciones individuales con un carácter reactivo y terapéutico, enfocadas a la resolución de problemas puntuales. En la orientación vocacional es primordial que la persona se reconozca así mismo, realizando un buen proyecto de vida y un autoconocimiento, desde temprana edad hasta que sea capaz de buscar nuevos aprendizajes significativos que le permitan estructurar mejor el carácter y la personalidad para estimular el desarrollo de sus características positivas. También se reconoce a la orientación vocacional como un medio para determinar cuál es su orientación profesional, a través de una asesoría psicológica, en la cual se debe brindar información del mundo, además del reconocimiento de sí mismo, siendo un proceso continuo donde la persona se motiva y aporta en la toma de su decisión teniendo en cuenta su quehacer.

Se establece que en la Orientación Vocacional profesional existen varios Psicólogos quienes dan importancia a las premisas que dicen que las personas son seres activos que buscan adaptarse a su ambiente y da mayor importancia a las influencias culturales donde de acuerdo a él, el individuo puede elegir su área vocacional y realmente como personas activas buscan adaptarse al medio, es así que a primera instancia la orientación vocacional pretende constituir personas que se adapten al lugar donde se encuentran y a partir de esto puedan desarrollar sus potencialidades. 


\section{REFERENCIAS BIBLIOGRAFICAS}

Alcívar, W. R. M., Santana, J. A. G., Zambrano, N. L. Z., Sardi, G. S., \& Riverón, J. L. N. (2020). Propuesta de guía para favorecer la orientación profesional para su ingreso a la educación superior ecuatoriana. Polo del Conocimiento: Revista científico-profesional, 5(10), 903-918.

Alvarez de Sayas, C. M. (2016) p.5. : La Escuela en la vida, décima edición, ALSIE Consultores Pedagógicos S. R. L., La Habana.

Carmona, B., Cortina, Y., \& Diana, M. (2012). La orientación profesional desde la perspectiva histórico cultural del desarrollo humano. ENTELEQUIA: Revista interdisciplinar , 55-63.

Centro de Investigación y Documentación Educativa. (2009) Orientación educativa: fundamentos teóricos, modelos institucionales y nuevas perspectivas. Madrid: OMAGRAF S. L.

Chul Han, B. (2017). La sociedad del cansancio. Barcelona: Herder Editorial.

Donoso-Vázquez, T., \& Sánchez Martí, A. (2013). Orientación educativa y profesional: estudio de caso. Barcelona.

FELDMAN, Robert (2007) Desarrollo Ppicológico. Pearson Educación, México.

Flores Delgado, A. (2021). LA PARTICIPACIÓN DE LOS PADRES DE FAMILIA EN LA CONSTRUCCIÓN DE SU PROYECTO DE VIDA DE LOS ESTUDIANTES DEL TERCER Y CUARTO AÑO DE LA INSTITUCIÓN EDUCATIVA SECUNDARIA GRAN UNIDAD ESCOLAR SAN JUAN BOSCO -SALCEDO -2018 [Universidad Nacional del Altiplano]. In Universidad Nacional del Altiplano. http://repositorio.unap.edu.pe/handle/UNAP/14748.

Gallegos Navas, M. M. (2017). Las familias de estudiantes con discapacidad en la escuela, sus necesidades y demandas. Caso Ecuador. Alteridad. Revista de Educación, 12(1), 20. https://doi.org/10.17163/alt.v12n1.2017.02.

Gómez, L., \& Palma, M. (2019). Caracterización de la generación Z y proyectos de vida.

Hervas Avilés, R. M. (2006). Orientación e intervención psicopedagógica y proceso de cambio. Granada: Grupo Edutorial Universitario (GRANADA).

JARRIN, P. (2002) Desarrollo Vocacional y Profesional. Don Bosco, Quito,

Lenzi, A., Borzi, S., \& Tau, R. (2010). El concepto de desarrollo en psicología: entre la evolución y la emergencia. Fundamentos en humanidades $N^{\circ}$ II, 137-161.

LEVIS, R. Aiken (2003). Test Psicológicos y Evaluación. Pearson Educación. México.

Lomelí-Parga, A., López-Padilla, M. y Valenzuela-González, J. (2016). Autoestima, motivación e inteligencia emocional: Tres factores influyentes en el diseño exitoso de un proyecto de vida de jóvenes estudiantes de educación media. Revista Electrónica Educare, 20(2), 1-22. doi: http://dx.doi.org/10.15359/ree.20-2.4 
Maree, J.G. (2018). Promoting Career Development and Life Design in the Early Years of a Person's Life. Early Child Development and Care, 188(4), 425-436.

http://dx.doi.org/10.1080/03004430.2017.1345892

Martín, M. (1996). Sistema experto de orientación vocacional-profesional (Un procedimiento informatizado de ayuda). . Sistema experto de orientación vocacional-profesional (Un procedimiento informatizado de ayuda). Madrid, Madrid, España.

Maura, V. G. (2003): «La Orientación Profesional desde la perspectiva histórico-cultural del desarrollo humano», Revista Cubana de Psicología, vol. 20, n. ${ }^{\circ} 3, \quad$ pp. 260-268, < 260-268, $<$ http://pepsic.bvsalud.org/pdf/rcp/v20n3/12.pdf > (2019-01-25).

Mendoza, I. G., Machado, E. F., \& Montes Oca, N. (2016). La estrategia de orientación vocacional y la elaboración de proyectos personales de vida / Vocational guidance strategy and personal life project design. Transformación, 122-133.

Ministerio de Educación del Ecuador. (2018). Herramientas para orientar la construcción de proyectos de vida de estudiantes. Quito: MinEduc.

Ministerio de Educación del Ecuador. (2015). Manual de orientación vocacional y profesional para los Departamentos de Consejería Estudiantil. Quito: MinEduc.

Ministerio de Educación del Ecuador. (2016). Modelo de Funcionamiento de los Departamentos de Consejería Estudiantil. Obtenido de https://educacion.gob.ec/ wpcontent/uploads/downloads/2017/05/ModeloFuncionamiento-DECEs.pdf

Moje Mayorca, D., \& Woolcott Oyague, O. (2018). El daño al proyecto de vida: Noción, estructura y protección jurídica según los parámetros establecidos por la Corte Interamericana de Derechos Humanos -CIDH. / The Harm Done to the Life Plan: Notion, Structure and Legal Protection According to the Param. Utopía y Praxis Latinoamericana, 23(1), 128-138. https://doi.org/10.5281/zenodo.1800841.

Pérez, N., Filella, G., \& Bizquerra, R. (2009). A los 100 años de la orientación: de la orientación profesional a la orientación psicopedagógica. Qurriculum $\mathrm{N}^{\circ} 22,55-71$.

Ruiz, J. R. (2011). Proyecto de vida, relatos autobiográficos y toma de decisiones : Lifestyle project, autobiographic narration and decision making process. Teoría y Praxis Investigativa, 6(1), 27. http://mendeley.csuc.cat/fitxers/d5ec96d7ca2a7c5c7931b8f0324fdbe.

Santana Sardi, G. A., \& Vigueras Moreno, J. A. (2019). Hacia un Sistema Virtual de orientación vocacional. Revista Cubana de Educación Superior, 38(3). P. 902.

Urquijo, M. J. (2014). La teoría en las capacidades de Amartya Sen. EDETANIA N 46, 63-80.

Ribiero, Marcelo; Uvaldo, María. (2007). Frank Parsons: Trajetória do pioneiro da orientação vocacional, profissional e de carreira. Revista Brasileira de Orientação Profissional, 19-31.

Rodríguez Beltran, M. (2009). Empoderamiento y promoción de la salud. Red de Salud N9, 21-31 